\title{
NO signaling and S-nitrosylation regulate PTEN inhibition in neurodegeneration
}

\author{
Young-Don Kwak', Tao Ma ${ }^{1,3}$, Shiyong Diao', Xue Zhang ${ }^{2}$, Yaomin Chen², Janet Hsu², Stuart A Lipton ${ }^{2,4}$, \\ Eliezer Masliah", Huaxi $\mathrm{Xu}^{2}$, Francesca-Fang Liao ${ }^{1 *}$
}

\begin{abstract}
Background: The phosphatase PTEN governs the phosphoinositide 3-kinase (PI3K)/Akt signaling pathway which is arguably the most important pro-survival pathway in neurons. Recently, PTEN has also been implicated in multiple important CNS functions such as neuronal differentiation, plasticity, injury and drug addiction. It has been reported that loss of PTEN protein, accompanied by Akt activation, occurs under excitotoxic conditions (stroke) as well as in Alzheimer's (AD) brains. However the molecular signals and mechanism underlying PTEN loss are unknown.

Results: In this study, we investigated redox regulation of PTEN, namely S-nitrosylation, a covalent modification of cysteine residues by nitric oxide ( $\mathrm{NO}$ ), and $\mathrm{H}_{2} \mathrm{O}_{2}$-mediated oxidation. We found that $\mathrm{S}$-nitrosylation of PTEN was markedly elevated in brains in the early stages of $A D(\mathrm{MCl})$. Surprisingly, there was no increase in the $\mathrm{H}_{2} \mathrm{O}_{2}{ }^{-}$ mediated oxidation of PTEN, a modification common in cancer cell types, in the MCI/AD brains as compared to normal aged control. Using several cultured neuronal models, we further demonstrate that S-nitrosylation, in conjunction with NO-mediated enhanced ubiquitination, regulates both the lipid phosphatase activity and protein stability of PTEN. S-nitrosylation and oxidation occur on overlapping and distinct Cys residues of PTEN. The NO signal induces PTEN protein degradation via the ubiquitin-proteasome system (UPS) through NEDD4-1-mediated ubiquitination.

Conclusion: This study demonstrates for the first time that NO-mediated redox regulation is the mechanism of PTEN protein degradation, which is distinguished from the $\mathrm{H}_{2} \mathrm{O}_{2}$-mediated PTEN oxidation, known to only inactivate the enzyme. This novel regulatory mechanism likely accounts for the PTEN loss observed in neurodegeneration such as in AD, in which NO plays a critical pathophysiological role.
\end{abstract}

\section{Background}

PTEN, the phosphatase and tensin homologue deleted on chromosome 10, is one of the most frequently mutated tumor suppressors in human cancers. The major, and best characterized, function of PTEN is its lipid phosphatase activity which dephosphorylates PIP3 to generate PIP2, and thus antagonizes the PI3K activity in the activation of Akt [1,2]. PTEN is expressed in almost all types of neurons [3] and is critical in multiple CNS functions such as neuronal differentiation and synaptogenesis [3-5], neuronal plasticity [6], neuronal injury (e.g., axonal branching/regeneration) $[7,8]$ myelin thickness of periphery nerves [9], and in drug

\footnotetext{
*Correspondence: fliao@uthsc.edu

'Department of Pharmacology, University of Tennessee Health Science Center, College of Medicine, 874 Union Avenue, Memphis TN, 38163, USA Full list of author information is available at the end of the article
}

addiction [10]. Our research has focused on elucidating novel roles for PTEN in neuronal death and neurodegeneration. We and others have reported that PTEN protein levels are reduced in AD brains, accompanied by elevated Akt phosphorylation [11-13]. We hypothesize that loss of PTEN protein is a key event regulating the PI3-K/Akt signaling, arguably the most important pro-survival pathway in neurons. In this study, we aimed to investigate the underlying molecular mechanism of PTEN loss.

Studies conducted in experimental models for cancer and diabetes have shown that PTEN regulation is rather complex. Multiple mechanisms might be involved in a decrease or loss of PTEN function, in addition to gene mutation and deletion. These mechanisms may include transcription and post-translational modifications (PTMs) which include phosphorylation, acetylation,

\section{() Biomed Central}


oxidation and ubiquitination [14]. PTEN is a relatively stable protein but its stability can be reduced in certain conditions, such as zinc treatment in neurons [15]. Phosphorylation at the S/T380-385 and T366/S370 sites influences PTEN stability as well as negatively regulating its enzymatic activity [16,17]. Besides phosphorylation, the ubiquitin-mediated proteasomal pathway is also an important mechanism regulating PTEN protein stability. We recently identified NEDD4-1 as the first ubiquitin ligase (E3) for PTEN that regulates PTEN degradation in multiple cancer types and in neurons $[15,18]$.

PTEN can be acutely regulated by oxidative stress and by endogenously produced reactive oxygen species (ROS) [19]. Oxidation of the active site cysteine residue(s) by ROS has long been recognized as a common mechanism regulating several key members of the protein tyrosine phosphatases (PTPs) including PTEN. A number of ROS species, including hydrogen peroxide $\left(\mathrm{H}_{2} \mathrm{O}_{2}\right)$, superoxide, peroxynitrite and nitrosothiol, modify PTEN on the critical cysteine residue (C124) and inactivate its lipid phosphatase activity in multiple cancer cell lines [20-24]. To investigate the molecular mechanism(s) underlying PTEN loss in the brains of AD patients, we examined these oxidative events with a special focus on $\mathrm{H}_{2} \mathrm{O}_{2}$ and NO-mediated S-nitrosylation; the later, a process of reversible addition of NO to Cyssulfur in proteins, has emerged as a major regulatory mechanism in fine-tuning many critical molecules in the neuronal death pathway and neurodegeneration [24]. To our surprise, only NO-mediated events lead to PTEN protein degradation, though both modifications inactivate PTEN's lipid phosphatase activity in neurons. To our knowledge, this is the first report of NO being the upstream signal that leads to a series of PTMs regulating PTEN protein degradation.

\section{Results}

S-nitrosylated PTEN levels are increased in MCI/AD brains, correlating with reduced PTEN and elevated P-Akt

Initially, we sought to investigate whether S-nitrosylated PTEN (SNO-PTEN) is produced in neurodegenerative disorders associated with high levels of nitrosative stress such as stroke, AD and Parkinson's disease (PD). We included in our tests those specimens taken from autopsy patients diagnosed at an early stage of $\mathrm{AD}$, called mild cognitive impairment (MCI), and compared them to aged matched control brain specimens (i.e., patients died from disorders not related to CNS). The patient cohort and information are summarized in the table [Additional file 1].

From semi-quantitative profiling of PTEN/Akt in 27 human brains, we found that SNO-PTEN was markedly induced in the entorhinal cortices, the most vulnerable region in $\mathrm{AD}$ brains, as early as the $\mathrm{MCI}$ stage but was nearly undetectable in age-matched normal brains (NC) (Figure 1A). Although the number of specimens with matching ages is too small to conduct serious statistical analysis, SNO-PTEN was elevated in all the Lewy body and PD brains as examined by biotin-switch assays, suggesting nitrosylation of PTEN is a common denominator in these neurodegenerative disorders. Due to the sporadic nature of $\mathrm{AD}$, it is not surprising that the level of SNO-PTEN is not inversely correlated with the level of PTEN protein in each individual case. However, statistical analysis reveals a trend (Figure 1B). Most significantly, the P-Akt was increased in the MCI samples compared to NC (Figure 1C) which correlates better with the elevated SNO-PTEN but not with the reduced PTEN protein in AD stage. Further analysis using Pearson's correlation analysis method revealed that SNO-PTEN is highly positively correlated with p-Akt level (0.8992) while PTEN level is negatively correlated with p-Akt (-0.6387) in AD samples. The inverse correlation between PTEN protein levels and P-Akt (Ser473) appears to be more significant for $\mathrm{AD} / \mathrm{NC}$ than for $\mathrm{MCI} / \mathrm{NC}$, suggesting that SNO-PTEN may not cause immediate protein degradation. However, the great variation of total Akt levels among the AD samples makes it difficult for a solid conclusion. Interestingly, the two AD cases (indicated by black asterisks) showing the highest levels of SNO-PTEN were also affected by cerebral infarcts (strokes); the two AD cases marked by red asterisks showed very low levels or complete loss of PTEN and low levels of Akt protein but relatively high levels of phosphorylated P-Akt. Surprisingly, we did not observe a significant change in the oxidative status of PTEN between $\mathrm{MCI}$ and $\mathrm{NC}$ sample groups (Figure 1D), as determined by a band-shift assay on non-reducing gels as described [22,23].

\section{PTEN can be S-nitrosylated in cultured neuronal cells by} exogenously and endogenously generated NO

Using cultured primary rat cortical neurons and biotinswitch assays, we found that SNO-PTEN can be rapidly induced in primary cultured cortical neurons treated with the physiological NO donor S-nitrosocysteine (SNOC) in a dose-dependent manner with detectable nitrosylation achieved by as little as $10 \mu \mathrm{M}$ SNOC and a plateau with $300 \mu \mathrm{M}$ (Figure 2A). This was also confirmed by a more quantitative fluorescent assay (DAN assay, Ref. [25]) using purified recombinant PTEN (Figure $2 \mathrm{~B}$ ). Additional neurotoxic compounds that induce $\mathrm{NO}$ generation, such as glutamate or $\beta$-amyloid peptides $\left(\mathrm{A} \beta_{25-35}\right)$ also induced robust SNO-PTEN within minutes (Figure 2C) and lasted for more than 10 hours, even after the NO donors were removed from the cultured media. Interestingly, the classic apoptotic stimuli, staurosporine/STS, did not induce SNO-PTEN. Since 


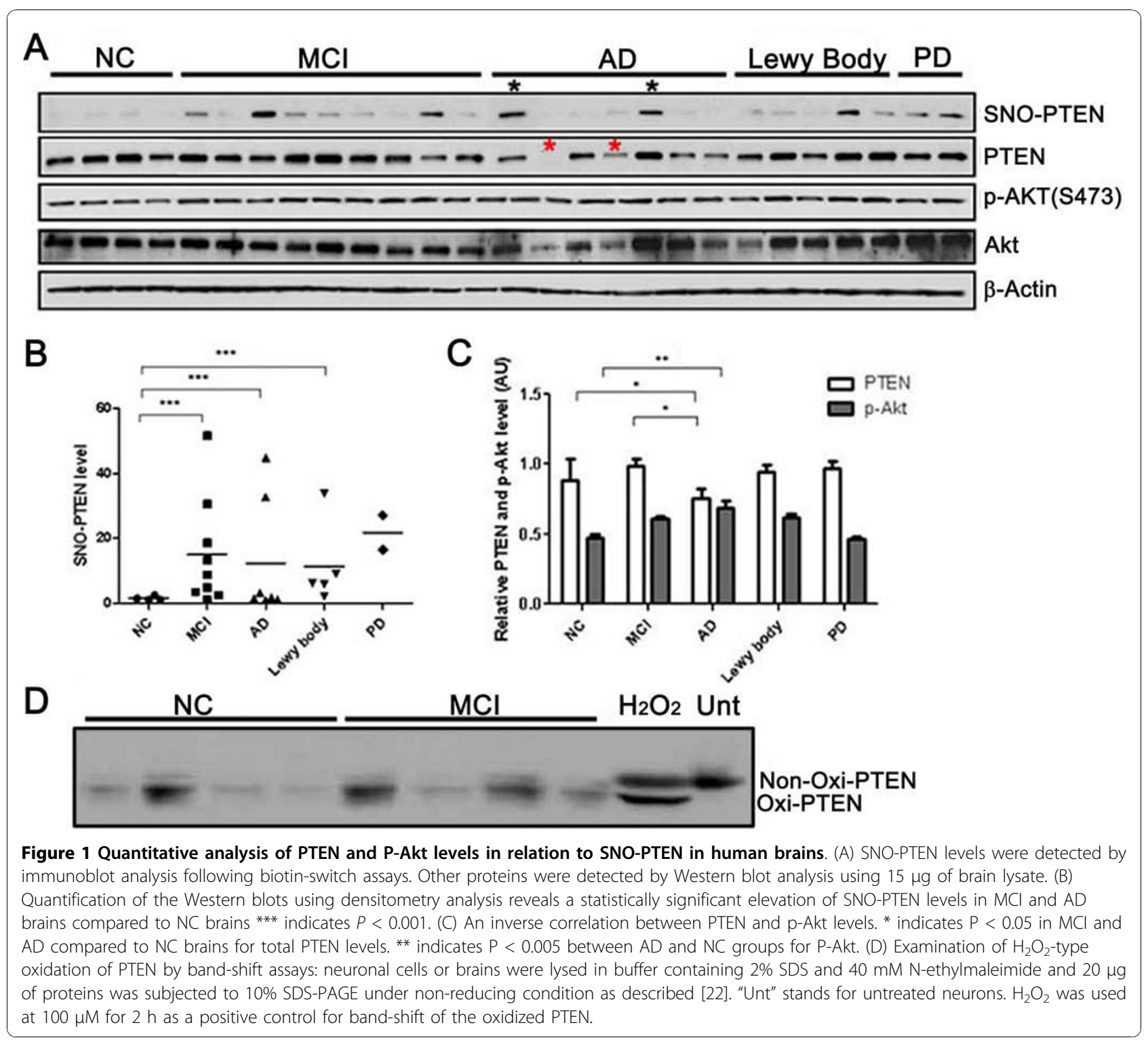

STS is known to activate $\mathrm{Ca}^{2+}$ influx through nonNMDAR type ion channels, our results indicate that the source of NO induced by glutamate/A $\beta$ is most likely via the activated nNOS in response to NMDARmediated $\mathrm{Ca}^{2+}$ influx. Two other mitochondrial ROS agents (rotenone and $\mathrm{MPP}^{+}$) also induced SNO-PTEN (data not shown); this is consistent with SNO-PTEN in PD brains and suggests that SNO-PTEN may play a common role in chronic degenerative diseases such as $\mathrm{AD}$ and $\mathrm{PD}$. In parallel, we found that after treatment of $\mathrm{SNOC/glutamate/A} \beta$, the majority of PTEN in neuronal cells $(>85 \%)$ remained unmodified by $\mathrm{H}_{2} \mathrm{O}_{2}$-type oxidation, indicating that $\mathrm{H}_{2} \mathrm{O}_{2}$ may not be the dominant oxidizing species induced by these treatments (Figure 2D). This is consistent with one recent report [26]. Furthermore, we found that the same experimental conditions that induced PTEN nitrosylation led to Akt activation as assessed by increased P-Akt; both were diminished by DTT treatment (Figure 2E).

NO signal induces subsequent enhanced ubiquitination of PTEN, leading to protein degradation via UPS

We found reduced PTEN ( $>50 \%)$ steady-state levels in neurons 2-4 h after exposure to SNOC, glutamate or $\mathrm{A} \beta_{25-35}$ peptides in a time-dependent manner (Figure $3 \mathrm{~A}$ and $3 \mathrm{~B}$ ). Interestingly, the reduction in PTEN was not observed after $\mathrm{H}_{2} \mathrm{O}_{2}$, staurosporine (STS), or okadaic acid (OA). The strong correlation between SNOPTEN formation and the reduced PTEN protein level suggests that the PTEN decrease may be a direct consequence of PTEN S-nitrosylation or may be caused otherwise by NO signaling. Indeed, pretreatment with a 


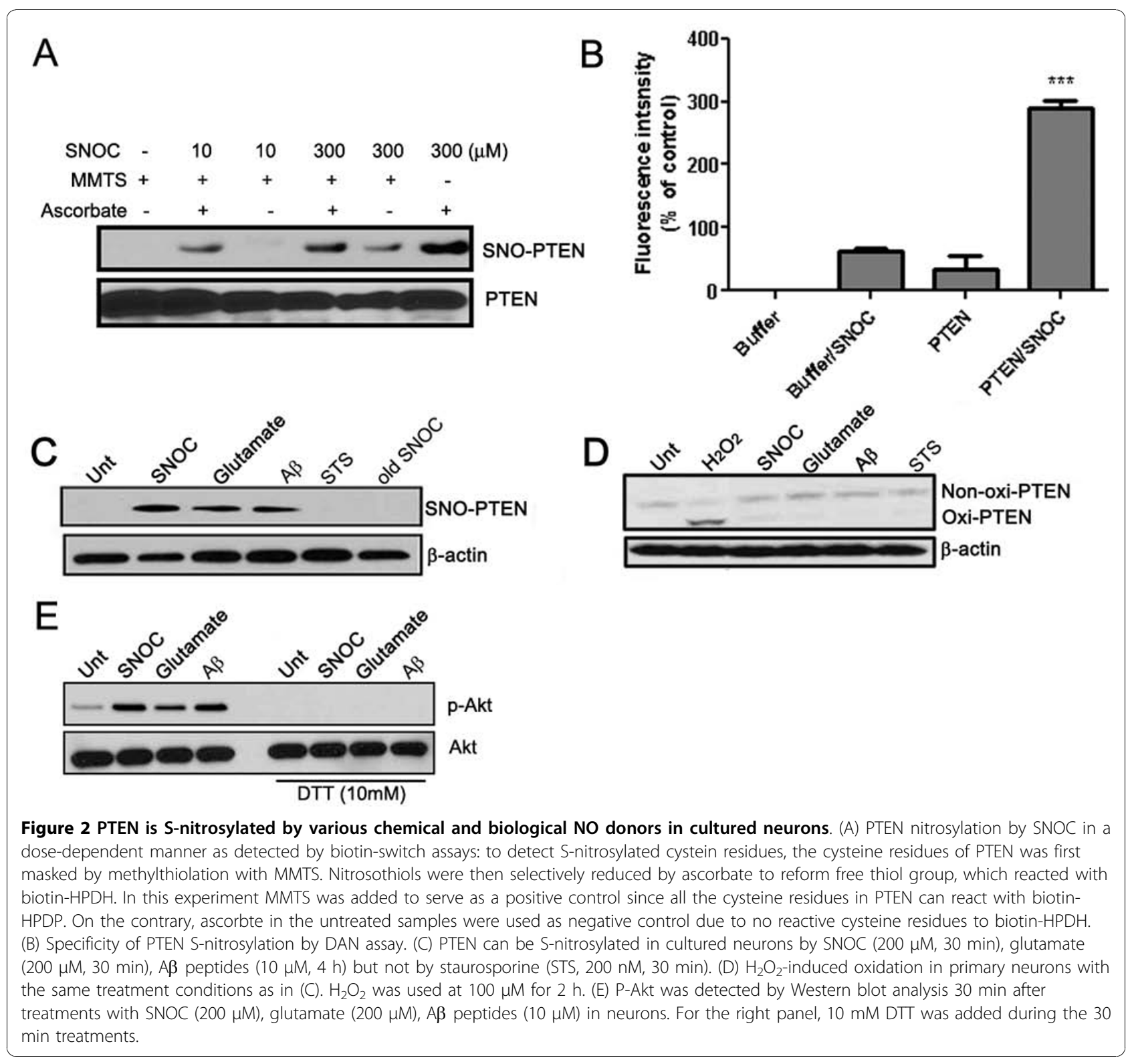

NOS inhibitor (l-NMMA) or the proteasome inhibitor MG132 for $5 \mathrm{~h}$ prior to glutamate exposure rescued the decrease in PTEN by over $70 \%$ and $50 \%$, respectively (Figure $3 \mathrm{C}$ ), suggesting protein degradation via the ubiquitin-proteasome system (UPS).

We also found that SNOC/glutamate/A $\beta$ all induce enhanced PTEN ubiquitination with the maximum effect seen $2 \mathrm{~h}$ after treatments (Figure 4A). Consistent with enhanced ubiquitination, we found increased physical interaction between PTEN and its E3 ligase NEDD41 after these treatments (Figure 4B), as we published recently [15]. Moreover, downregulation of NEDD4-1 by its specific siRNA prevented PTEN degradation induced by SNOC treatment (Figure $4 \mathrm{C}$ ). Together with the
MG132 data, these findings indicate the involvement of the UPS in PTEN protein degradation. It is not clear whether S-nitrosylation of PTEN itself can lead to enhanced PTEN ubiquitination and protein degradation.

SNO-PTEN likely occurs at multiple Cys residues in the phosphatase domain of PTEN, inactivating its lipid phosphatase activity

We next examined the effect of PTEN S-nitrosylation on the lipid phosphatase activity of PTEN. By conventional Malachite Green assay using PIP3 as substrate [2], SNOC treatment inactivated PTEN in a dose-dependent manner with $80 \%$ of activity lost at $400 \mu \mathrm{M}$, which was reversed by DTT (Figure 5). To determine the target 

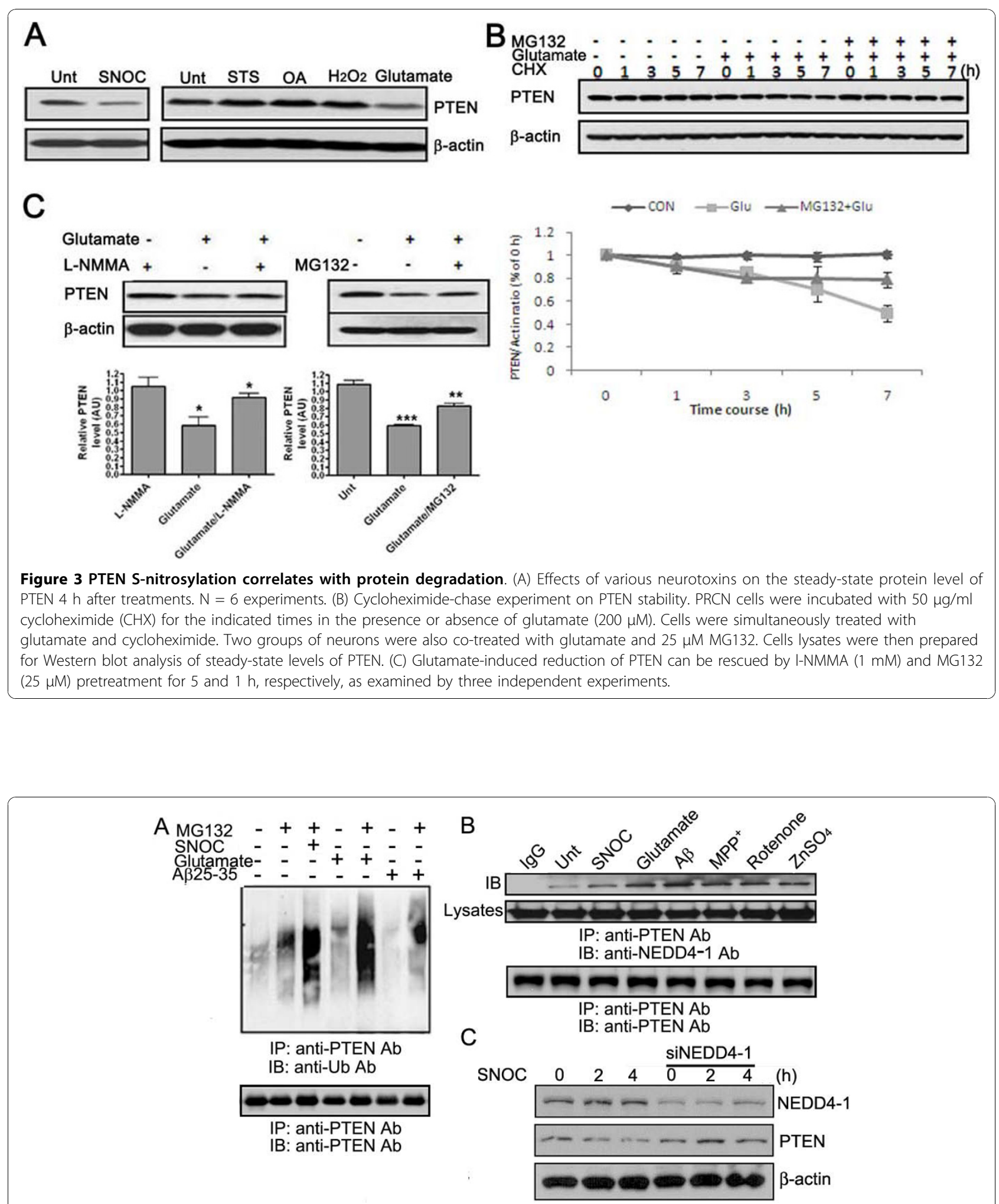

Figure 4 NO signals induce enhanced ubiquitination of PTEN, leading to protein degradation. (A) Treatments with SNOC, glutamate or A $\beta$ peptides increase PTEN ubiquitination, as determined by IP-Western analysis. (B) Enhanced physical interaction between PTEN and NEDD4-1 upon various treatments at the conditions used in other experiments, as determined by co-immunoprecipitation/Western blot analysis. Figure is chosen as the representative of three independent experiments. (C) Downregulation of NEDD4-1 by siRNA ( $4 \mu \mathrm{g}$ ) prevents PTEN protein degradation upon SNOC treatment. 

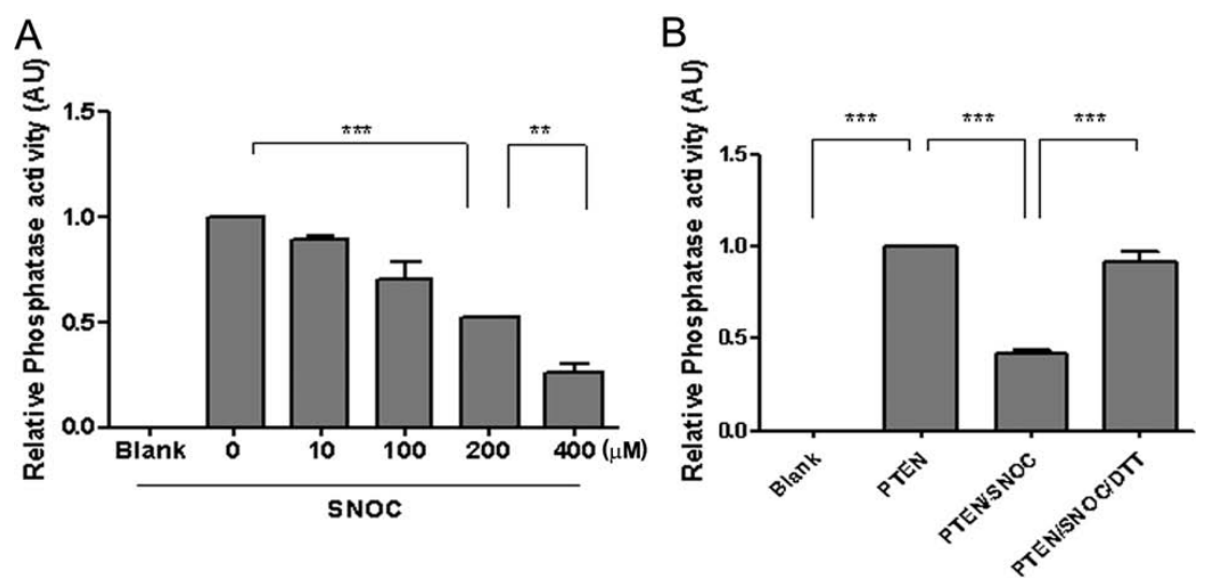

Figure 5 SNOC treatment inactivates PTEN's lipid phosphatase activity. (A) Dose-dependent effect of SNOC on lipid phosphatase activity as determined by Malachite Green assay. Data presented are means based on five independent experiments. ** indicates $p<0.05$ and ${ }^{* * *}$ indicates $p<0.005$. (B) DTT treatment $(10 \mathrm{mM})$ completely restores the PTEN's lipid phosphatase activity abolished by SNOC $(300 \mu M)$.

site(s) of SNOC on PTEN, we performed site-directed mutagenesis on each of the 10 cysteine residues (Cys to Ala replacement) (Figure 6A). Using biotin-switch assays, we examined the nitrosylation levels of each PTEN mutant after transfection into mouse neuroblastoma N2a cells 30 min after exposure to $200 \mu \mathrm{M}$ SNOC and compared SNO-PTEN levels to those of WT PTEN. For the 10 PTEN mutants, only Cys83A mutant displayed significantly reduced S-nitrosylation $[75 \%$ reduction based on the mean values of 7 experiments, Figure $6 \mathrm{~B}$ and also see additional file 2]. Thus, the most likely candidate cysteine residue that is physiologically S-nitrosylated is C83. The C71A and C124A mutations only partially affected S-nitrosylation $(\sim 25 \%)$, and the double mutant $\mathrm{C} 71 \mathrm{C} 83 \mathrm{~A}$ was nitrosylated similarly to the C83A mutant. The C71C124A double mutant had the same effect as single mutants. In contrast, triple mutant C71C83C124A completely abolished the PTEN nitrosylation, suggesting an involvement of all three Cys residues to varying degrees under our conditions. Furthermore, we also observed greatly reduced ubiquitination for the triple mutant (Figure 6C) which suggests the possibility that its ubiquitination may be partially dependent on PTEN nitrosylation. Interestingly, the steady-state levels of the triple mutant was not reduced by SNOC treatment as compared to WT or C71C83 double mutant [see additional file 3], indicating that the C124 residue is a critical determinant for PTEN protein stability.

\section{Downregulation of PTEN and elevated P-Akt levels protect neurons against $A \beta$-induced toxicity}

In cultured primary neurons, exposure to $A \beta$ or NMDA induced a rapid decrease in PTEN and increase in P-Akt levels. Downregulation of endogenous PTEN via specific siRNA produces neuroprotection, as evidenced by preserved neuronal structures (Figure 7A and 7B; MAP2/NeuN staining/Green). Moreover, the frontal region of the brain of PTEN heterozygous mice bear reduced PTEN as compared to WT littermate controls $(\sim 50 \%$ reduction) and increased basal P-Akt levels (Figure $7 \mathrm{C}$ ). Cortical neurons from PTEN+/- brains exhibited increased resistance to $A \beta$-induced cell death compared to those from WT littermate controls (Figure 7D), suggesting a neuroprotective role for PTEN downregulation.

\section{Discussion}

PI3K/Akt is arguably the most important cell-survival signaling pathway for neurons. As the key negative regulator of the PI3K/Akt pathway, PTEN is an important target of study for neuroprotection during neurodegeneration. In this work, we are the first to demonstrate NO-mediated redox regulation as the mechanism of PTEN protein degradation. We also demonstrate that NO rapidly induces S-nitrosylation of PTEN, thereby inactivating it. Moreover, NO, but not $\mathrm{H}_{2} \mathrm{O}_{2}$, induces PTEN protein degradation. Loss of PTEN protein is reportedly associated with myocardial and brain ischemia [27-29], presumably as a cellular adaptive stress response to activate the pro-survival PI3K/Akt signaling. Herein, we show that PTEN loss also occurs in neurons in response to a variety of neurotoxins (e.g., glutamate and $A \beta$ peptides) as well as in chronic neurodegenerative conditions such as AD and PD brains. Hence, our findings on NO-mediated PTEN protein degradation may represent a common mechanism underlying PTEN loss in these acute and chronic degenerative conditions, in which NO plays a critical pathophysiological role. 


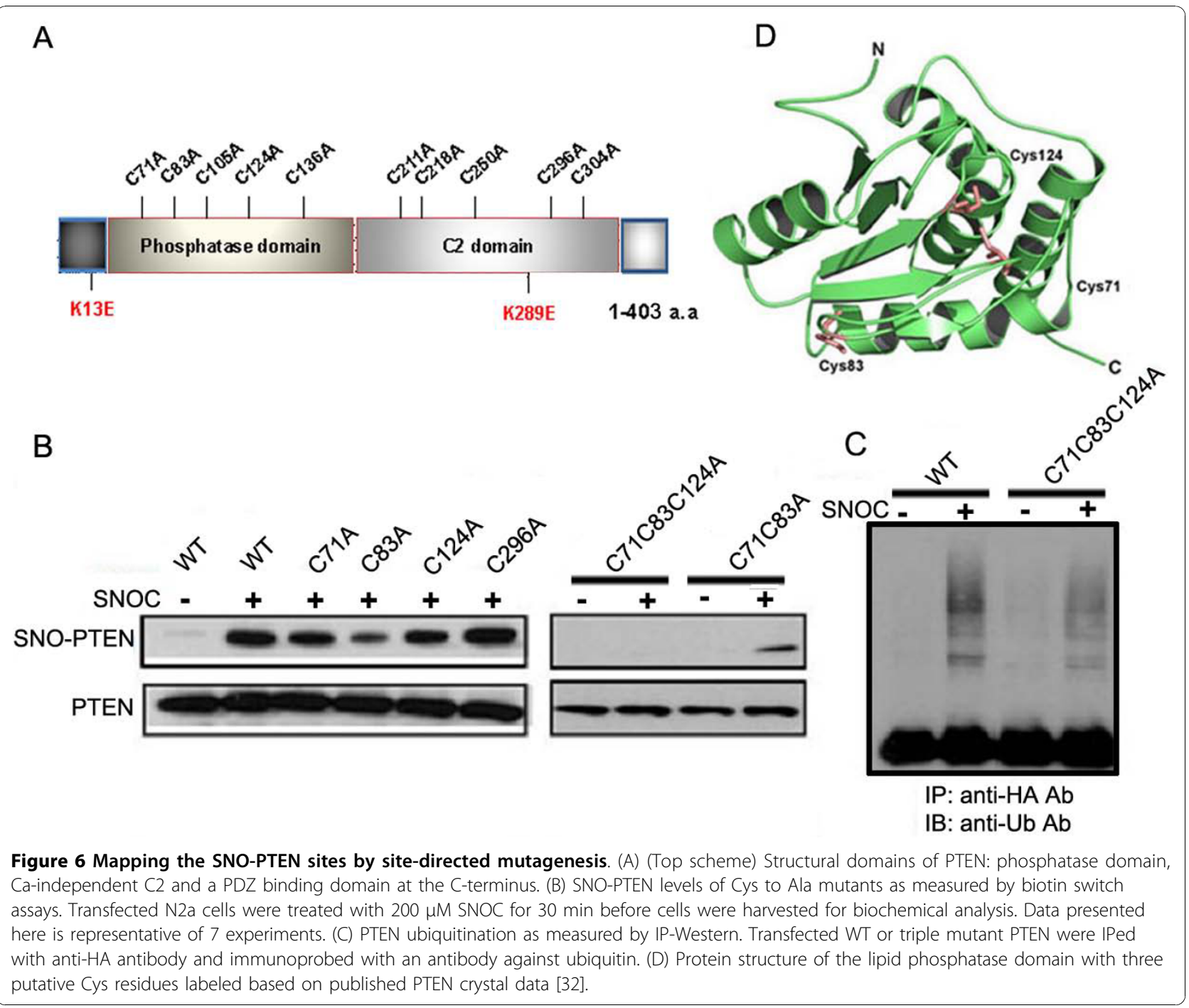

It is widely accepted that oxidative stress is one of the earliest changes that occurs in the pathogenesis of $\mathrm{AD}$, arising from the imbalance between increased production of reactive oxygen and nitrogen species and impaired antioxidant defenses, as reflected in the accumulation of oxidative damage to macromolecules detected in $\mathrm{MCI}$, the clinical precursor of $\mathrm{AD}$, and $\mathrm{AD}$ brains [30,31]. $\mathrm{H}_{2} \mathrm{O}_{2}$-induced modification and S-nitrosylation represent the two dominant oxidative events through targeted modifications of critical Cys residues in proteins. $\mathrm{H}_{2} \mathrm{O}_{2}$-induced PTEN oxidation was reported to cause the formation of an intra-chain disulfide bond between C71-124 [22], and is reflected in faster mobility species by band-shift assay on non-reducing gels (Figure 2D). SNOC did not induce a band shift of PTEN on non-denaturing gels and thus unlikely induced a major conformational change due to intra-chain disulfide bond formation. However, our mutagenesis data suggest the involvement of overlapping residues on $\mathrm{C} 71$ and $\mathrm{C} 124$. It is, therefore, reasonable to predict that these two oxidative modifications can compete with each other when both oxygen and NO species are present. Moreover, our data indicate that NO-mediated oxidation is the predominant form of PTEN in aging brains and in $\mathrm{MCI} / \mathrm{AD}$ brains (Figure 1D), which may generalize to other neurodegenerative diseases such as $\mathrm{PD}$.

Although our mutagenesis studies (Figure 6B) cannot determine the nitrosylated sites unambiguously, the results strongly suggest that C83 is likely the most significant physiological site of S-nitrosylation on PTEN. It is possible that multiple Cys residues are involved depending on the spatial and temporal concentrations of NO. It is well known that the $\mathrm{C} 124$ residue is critical for the enzymatic activity of PTEN [32]; mutation of this residue results in inactivation of both the protein and phospholipid lipase activities of PTEN. Our data [see 


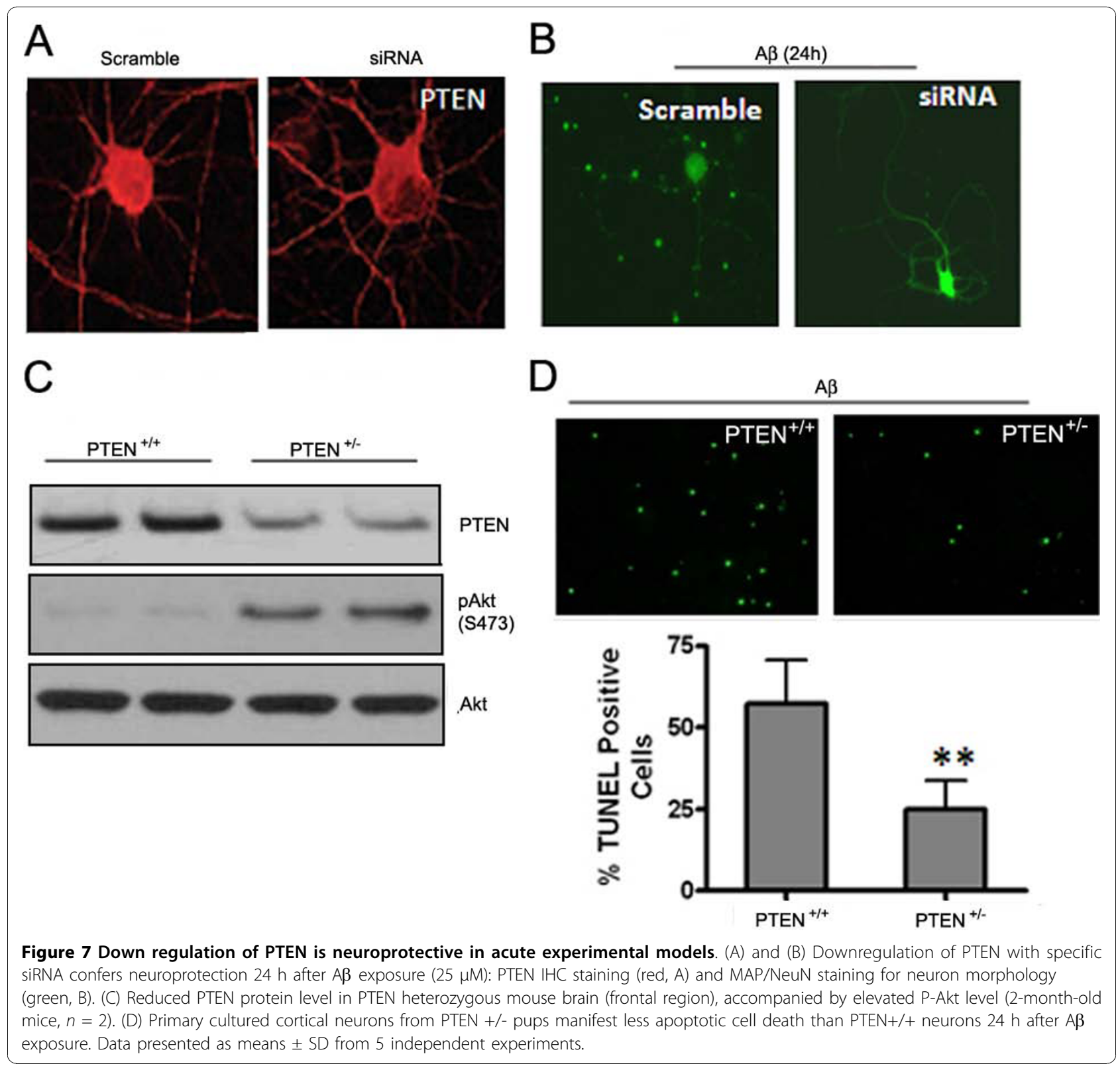

additional file 3] also indicate its importance in PTEN protein stability; mutation on this Cys residue renders PTEN resistance to SNOC-induced protein degradation. It warrants further investigation of the underlying mechanisms. Structural analysis based on the solved crystal structure of PTEN [33] indicates that Cys83 is not in close vicinity to $\mathrm{C} 124$, which is located face-toface to C71 (Figure 6D). It is therefore not yet clear mechanistically how C83, in conjunction with C71 and C124, affects the lipid phosphatase activity of PTEN.

Our results demonstrate NO-mediated PTEN protein degradation via UPS, as evidenced by enhanced ubiquitination. There are several precedents showing that protein S-nitrosylation can be functionally coupled to its ubiquitination and modulate protein degradation [34-38]. It is possible that S-nitrosylation of PTEN plays a direct causative role in its degradation, as evidenced by enhanced ubiquitination upon SNOC treatment (Figure 4A). Given the role of phosphorylation in modulating PTEN protein stability and activity previously revealed by cancer cell models [14,38], it is also possible that NO signal induces alteration on PTEN phosphorylation status which is the cause of enhanced ubiquitination and protein degradation. Interestingly, the two putative kinases identified as responsible for phosphorylation of the two major clusters on PTEN (Ser/Thr cluster $380 / 382 / 383$ and Thr366/Ser370), namely glycogen synthase kinase GSK3 $\beta$ and casein kinase CK2, are both 
implicated in neurodegeneration $[39,40]$. Moreover, our unpublished data show that OA can prevent PTEN dephosphorylation to a significant extent following exposure to SNOC, suggesting that PP2A, PP1 and perhaps PP2B, all major protein phosphatases implicated in AD [41], may play a role in modulating PTEN stability. Thus, the detailed interplay between kinases and phosphatases warrants further investigation.

Although we made our initial observation in MCI/AD brain samples, the loss of PTEN is also reported to occur in both myocardial [27] and cerebral ischemia/ reperfusion $[28,29]$. Therefore, we believe that PTEN inhibition in these acute conditions mediates subsequent activation of PI3K/Akt signaling, which is known to be key to the endogenous protective effect, similar to the neuronal adaptive response. Paradoxically, we found that loss of PTEN occurs in human brains with AD, accompanied by elevated P-Akt in AD-affected regions. Moreover, the elevated P-Akt has often been detected in the same neuron bearing neurofibrillary tangles, which is a major pathological hallmark of AD consisting of hyperphosphorylated tau protein [13]. Although a reduction of P-Akt was also reported by previous studies [42]; we repeatedly found elevated P-Akt levels in degenerating neurons in $\mathrm{AD}$ brains by immunohistochemistry (unpublished data), which is consistent with several other reports $[11,43]$. Taken with our earlier finding that downregulated PTEN resulted in tau hyperphosphorylation and aggregation, we speculate that the loss of PTEN may be a contributing factor to neurodegeneration over the course of the disease due to chronically or excessively activated Akt signaling, which we speculate to be detrimental, as has been reported in several other chronic diseases [44,45]. Akt is activated in samples from patients with chronic heart failure; biochemical analyses demonstrated that chronic Akt activation induces feedback inhibition [46]. This nascent theory appears to be supported by a finding that conditional PTEN ablation in the forebrain region which caused impaired synaptic structure and function with concomitant constitutive activation of Akt and mTOR signaling [47]. An alternative view would be that the increase in Akt signaling occurs in response to damage, as an adaptive response, but fails to reach significantly protective levels under these chronic conditions. Only future experiments will be able to distinguish between these alternatives.

\section{Conclusions}

In summary, our studies have demonstrated that S-nitrosylation of PTEN plays a major role in PTEN regulation in neuronal systems and that NO signaling induces S-nitrosylation and ubiquitination to modulate both PTEN protein degradation and enzymatic activity, which may represent the underlying regulatory mechanism of the PI3K/Akt signaling pathway in both acute and chronic neurodegeneration. A fuller understanding of the molecular mechanisms underlying the cascade of events leading to PTEN inhibition in both acute and chronic settings will advance our knowledge of PTEN regulation in the CNS. It may also be instrumental in future therapeutic design of novel target-based intervention in treating neurodegenerative diseases and perhaps other age-related conditions such as cancers, diabetes, cardiovascular ischemia and stroke.

\section{Methods}

\section{Cell culture, treatment of neurotoxic reagents} and transfection

Primary cultured cortical neurons (PRCN) and neuroblastoma N2a cells were prepared as described [48]. For the majority of experiments, freshly prepared SNOC (Sigma-Aldrich, St. Louis, MO) was added at $200 \mu \mathrm{M}$ to 2 -week-old cultured neurons for $30 \mathrm{~min}$. SNOC was freshly prepared as described [49]. In brief, to prepare a $100 \mathrm{mM}$ stock solution, $0.0069 \mathrm{~g}$ sodium nitrite and $0.0121 \mathrm{~g}$ l-cysteine were added to $950 \mu \mathrm{l} \mathrm{H}_{2} \mathrm{O}$. Then, $50 \mu \mathrm{l}$ of $10 \mathrm{~N} \mathrm{HCl}$ is added to adjust $\mathrm{pH}$ to be 7.4. For glutamate (Sigma-Aldrich), A $\beta_{25-35}$ peptides (Bachem, Torrance, CA), and staurosporine/STS (Sigma-Aldrich), neurons were treated for 4 hours before cell lysates were prepared. For suppression of NOS activity, l-NMMA (Sigma-Aldrich, $1 \mathrm{mM}$ ) was applied to neurons before exposure to neurotoxic reagents.

Transient transfections were performed with plasmid constructs for pEF-PTEN-WT (kindly provided by Dr. Hong Wu, UCLA, CA) and its site-directed mutagenized constructs (Cysteine to Alanine substitution).

\section{Human patient brains}

Human brain samples were provided by UCSD, San Diego, CA and were analyzed with institutional permission under California and National Institutes of Health guidelines. Informed consent was obtained following the procedures of the Institutional Review Boards of the Sanford-Burnham Institute for Medical Research.

\section{Biotin-switch assay for detection of PTEN S-nitrosylation}

A biotin switch assay for detection of SNO-PTEN was performed as previously described with minor modification [50]. PRCN cultures were exposed to various concentrations $(10,50,100,200$ and $300 \mu \mathrm{M})$ of SNOC and old SNOC for $30 \mathrm{~min}$, glutamate, STS, or $\mathrm{A} \beta_{25-35}$ for $4 \mathrm{~h}$.

\section{Fluorometric measurement of S-nitrosylation of PTEN}

S-nitrosylation of PTEN was measured as previously described [25]. 
Detection of $\mathrm{H}_{2} \mathrm{O}_{2}$-mediated oxidation-band shift assay Cells were lysed in buffer containing 2\% SDS and 40 $\mathrm{mM} \mathrm{N}$-ethylmaleimide and $20 \mu \mathrm{g}$ of protein was subjected to $10 \%$ SDS-PAGE under non-reducing condition as described $[22,23]$.

\section{PTEN phosphatase assay-Malachite green assay}

Recombinant PTEN, which was expressed in baculovirus, was assessed for the phosphatase activity as previously described [2]. Enzymatic activity of PTEN was quantified as activity measured relative to the control.

\section{Immunoprecipitation and Western blot analysis}

IP-Western experiments were performed as described [48]. The following antibodies (Abs) were used: rabbit anti-PTEN Ab (Cell Signaling, Danvers, MA, USA); goat anti-PTEN (Santa Cruz, Santa Cruz, CA); mouse anti- $\alpha$ tubulin Ab (Sigma, St. Louis, MO, USA); rabbit anti-PAkt Ab (Cell Signaling, Danvers, MA, USA); rabbit Akt 1/2/3 (Santa Cruz, Santa Cruz, CA); mouse anti-monopoly-Ub Ab (Enzo, Plymouth Meeting, PA); rabbit antiNEDD4 Ab (Upstate, Charlottesville. VA, USA); mouse anti- $\beta$-actin Ab (Sigma); anti-mouse IgG and anti-rabbit IgG horseradish peroxidase-conjugated Abs (Chemicon, Temecula, CA, USA).

\section{Cycloheximide chase assay}

PRCN cells were incubated with $50 \mu \mathrm{g} / \mathrm{ml}$ cycloheximide (Sigma, St. Louis, MO, USA) for the indicated times in the presence or absence of glutamate (200 $\mu \mathrm{M})$. Cells were simultaneously treated with glutamate and cycloheximide. To examine whether the UPS is involved in nitrosative stress induced PTEN reduction, cells were co-treated with glutamate and $25 \mu \mathrm{M}$ MG132. Cells lysates were then prepared for Western blot analysis.

\section{Sindbis virus-delivered RNA interference mediated silencing of PTEN in PRCN}

Three different sets of siRNAs were designed by Ambion for rat PTEN (RefSeq NM_031606, Ambion). The annealed oligonucleotides encoding siRNAs were cloned into the pIRES-enhanced RFP (Invitrogen) using EcoRI/BamHI sites and the resulting siRNAIRES-RFP fragment was further cloned into pSinRep5 (Invitrogen). The efficiency and specificity of PTEN-siRNAs were then assessed by RT-PCR and western blot analysis. Virus particles were generated and infection performed in primary neurons according to the manufacturer's protocol as described previously [13].

\section{Site-directed mutagenesis of PTEN}

PTEN mutants were created by site-directed mutagenesis (QuikChange ${ }^{\oplus}$ II Site-Directed Mutagenesis Kits, Stratagene, La Jolla USA) at C71, C83, C105, C124, C136, C211, C218, C250, C296, C304, and its double/ triple mutants with various combinations. The primers for C71A forward 5'-TTTAAAGCATAAAAACCATTA CAAGATATACAA-3' and reverse 5'-GTCATAATGTC TAGCAGCAAGATTGTATAT-3'; C83A forward 5'GACACCGCCAAATTTAATGCCAGAGTTGCACAA3' and reverse 5'-GATATTGTGCAACTCTGGCAT TAATGGCGG-3'; C105A forward 5'-GAACTTAT CAAACCCTTTGCTGAAGATCTTGAC-3' and reverse 5'-CCATTGGTCAAGATCTTCAGCAAAGGGTTTG AT-3'; C124A forward 5'-CATGTTGCAATTCACGCT AAAGCTGGAAAG-3' and reverse 5'-GTCCCTTT CCAGCTTTGCGTGAATTGCTGGAATTGCTGCAA3'; C136A forward 5'-GACGAACTGGTGTAATGA TAGCCGCATATTTAT-3' and reverse 5'-CCCGATGA TATAAATATGCGGCTATCATTACAC-3'; C211A forward 5'-GTTCAGTGGCGGAACTGCCAATCCTCA GTTTG-3' and reverse 5'-CACAAACTGAGGATTGGCAGTTCCGCCACTGAA-3'; C218A forward 5'CCTCAGTTTGTGGTCGCCCAGCTAAAGGTGAA-3' and reverse 5'-CTTCACCTTTAGCTGGGCGACCAC AAACTGAGC-3'; C250A forward 5'-CAGCCGTTA CCTGTGGCTGGTGATATCAAAG-3' and reverse 5'CTTTGATATCACCAGCCACAGGTAACGGCTG-3'; C296A forward 5'-TCAGAAAAAGTAGAAAATGG AAGTCTAGCTGAT-3' and reverse 5'-CAAATGCTA TGGATTTCTTGATCAGCTAGACTT-3'; C304A forward 5'- CAAGAAATCAGCATTGCCAGTATAGA GCGT-3' and reverse 5'-CTGCACGCTCTATACTGG CAATGCTATCGATTT-3' were utilized for PCR amplification, respectively. PCR amplification was performed according to the company's protocol. Once single mutants were constructed, we used these mutants as a template for further construction of double/triple mutants.

Immunocytochemistry for neuronal morphology/dendritic structure and cell death (TUNEL) assays

IHC on PTEN (1:500) and MAP-2/NeuN (1:500/each) and apoptotic staining were performed as described [48] on two week-cultured neurons seeded on glass cover slips. In PTEN heterozygous mice were genotyped as described [51].

\section{Statistics}

All quantitative data were presented as means \pm SDV. Comparison between groups were analyzed with unpaired ANOVA using Graphpad PRIZM software (La 
Jolla, CA, USA) and values of $p<0.05$ were considered to be significant.

\section{Additional material}

\section{Additional file 1: The table of the patient brain information.}

Additional file 2: Additional data on the Cys mutants. SNO-PTEN levels were determined by biotin-switch assays and data were analyzed by densitometry of the SNO-PTEN/total PTEN ratio, indicating that the majority of C211-304 located in the C2 domain do not seem to be the direct sites of S-nitrosylation.

Additional file 3: Effect of double and triple Cys mutants on PTEN protein stability upon SNOC treatment. Various mutant plasmids were transiently introduced to N2a cells along with WT control and cells were treated with SNOC $(200 \mu \mathrm{M}) 48 \mathrm{~h}$ after transfection. The steady-state levels of PTEN were determined at various time points by Western blot analysis using anti-HA antibody to probe on the exogenously expressed PTEN.

\section{Acknowledgements}

We thank Dr. Dong-Hyung Cho for helpful discussion and Robert C. Thompson for excellent technical support. We also thank Drs. Xuejun Jiang and Wei Pan for providing recombinant PTEN protein. This work was partially supported by the NIH grants (R01 NS054880, AG031893 to F-F. L.; R01 AG021173, R01 NS046673 and R01 AG030197 to H.X., AG18440, AG5131, AG022074 to E. M., P01 ES016738 to S.A.L.), the grants from the Alzheimer's Association (to H.X. and F.-F.L.), and the American Health Assistance Foundation (to H.X.). Y-D K was the recipient of 2008 Young Scholar Award from Alzheimer's association San Diego/Imperial Chapter.

\section{Author details}

'Department of Pharmacology, University of Tennessee Health Science Center, College of Medicine, 874 Union Avenue, Memphis TN, 38163, USA. ${ }^{2}$ Del E. Webb Center for Neuroscience, Aging, and Stem Cell Research, Sanford-Burnham Medical Research Institute, 10190 North Torrey Pines Road, La Jolla, CA 92037, USA. ${ }^{3}$ Department of Neurology, Wuxi the Second People's Hospital, Affiliated to Nanjing Medical University, Jiangsu 214002 PR China. ${ }^{4}$ Department of Neurosciences, University of California at San Diego, 9500 Gilman Drive, La Jolla, CA 92039, USA.

\section{Authors' contributions}

Author contributions: FFL designed research; Y-DK, TM, S-YD, XZ, Y-MC, and JS performed experiments; SAL, EM, HX, analyzed data. FFL and Y-DK wrote the paper. All authors have read and approved the final manuscript.

\section{Competing interests}

The authors declare that they have no competing interests.

Received: 2 October 2010 Accepted: 10 November 2010 Published: 10 November 2010

\section{References}

1. Myers MP, Stolarov JP, Eng C, Li J, Wang SI, Wigler MH, Parsons R, Tonks NK: PTEN, the tumor suppressor from human chromosome 10q23, is a dualspecificity phosphatase. Proc Natl Acad Sci USA 1997, 94:9052-9057.

2. Maehama T, Dixon JE: The tumor suppressor, PTEN/MMAC1, dephosphorylates the lipid second messenger, phosphatidylinositol 3,4,5-trisphosphate. J Biol Chem 1998, 273:13375-13378.

3. Lachyankar MB, Sultana N, Schonhoff CM, Mitra P, Poluha W, Lambert S, Quesenberry PJ, Litofsky NS, Recht LD, Nabi R, Miller SJ, Ohta S, Neel BG, Ross AH: A role for nuclear PTEN in neuronal differentiation. I Neurosci 2000, 20:1404-1413.

4. Groszer M, Erickson R, Scripture-Adams DD, Lesche R, Trumpp A, Zack JA, Kornblum HI, Liu X, Wu H: Negative regulation of neuronal stem/ progenitor cell proliferation by the PTEN tumor suppressor gene in vivo. Science 2001, 294:2186-2189.
5. Backman SA, Stambolic V, Suzuki A, Haight J, Elia A, Pretorius J, Tsao MS Shannon P, Bolon B, Ivy GO, Mak TW: Deletion of PTEN in mouse brain causes seizures, ataxia and defects in soma size resembling LhermitteDuclos disease. Nat Genet 2001, 29:396-403.

6. Chang N, El-Hayek YH, Gomez E, Wan Q: Phosphatase PTEN in neuronal injury and brain disorders. Trends in Neurosci 2007, 30:581-586.

7. Park KK, Liu K, Hu Y, Smith PD, Wang C, Cai B, Xu B, Connolly L, Kramvis I, Sahin M, He Z: Promoting axon regeneration in the adult CNS by modulation of the PTEN/mTOR pathway. Science 2008, 322:963-966.

8. Drinjakovic J, Jung H, Campbell DS, Strochlic L, Dwivedy A, Holt CE: E3 ligase Nedd4 promotes axon branching by downregulating PTEN. Neuron 2010, 65:341-357.

9. Cotter L, Ozçelik M, Jacob C, Pereira JA, Locher V, Baumann R, Relvas JB, Suter U, Tricaud N: Dlg1-PTEN interaction regulates myelin thickness to prevent damaging peripheral nerve overmyelination. Science 2010, 328:1415-1418.

10. Ji SP, Zhang Y, Van Cleemput J, Jiang W, Liao M, Li L, Wan Q, Backstrom JR, Zhang X: Disruption of PTEN coupling with 5-HT2C receptors suppresses behavioral responses induced by drugs of abuse. Nat Med 2006, 12:324-329.

11. Griffin RJ, Moloney A, Kelliher M, Johnston JA, Ravid R, Dockery P, O'Connor R, O'Neill C: Activation of Akt/PKB, increased phosphorylation of Akt substrates and loss and altered distribution of Akt and PTEN are features of Alzheimer's disease pathology. J Neurochem 2005, 93:105-117.

12. Rickle A, Bodfanovic N, Volkmann I, Zhou X, Pei JJ, Winblad B, Cowburn RF: PTEN levels in Alzheimer's disease medial temporal cortex. Neurochem Int 2006, 48:114-123.

13. Zhang X, Li F, Bulloj A, Zhang YW, Tong G, Zhang Z, Liao FF, Xu H: Tumorsuppressor PTEN affects tau phosphorylation, aggregation, and binding to microtubules. FASEB J 2006, 20:1272-1274.

14. Wang X, Jiang X: Post-translational regulation of PTEN. Oncogene 2008, 27:5454-5463.

15. Kwak YD, Wang B, Pan W, Xu H, Jiang X, Liao FF: Functional interaction of phosphatase and tensin homologue (PTEN) with the E3 ligase NEDD4-1 during neuronal response to zinc. J Biol Chem 2010, 285:9847-9857.

16. Vazquez F, Ramaswamy S, Nakamura N, Sellers WR: Phosphorylation of the PTEN tail regulates protein stability and function. Mol Cell Biol 2000, 20:5010-5018.

17. Maccario H, Perera NM, Davidson L, Downes CP, Leslie NR: PTEN is destabilized by phosphorylation on Thr 366. Biochem J 2007, 405:439-444.

18. Wang X, Trotman LC, Koppie T, Alimonti A, Chen Z, Gao Z, Wang J, Erdjument-Bromage $H$, Tempst $P$, Cordon-Cardo C, Pandolfi PP, Jiang X: NEDD4-1 is a proto-oncogenic ubiquitin ligase for PTEN. Cell 2007, 128:129-139.

19. Leslie NR, Bennett $D$, Lindsay YE, Stewart H, Gray A, Downes CP: Redox regulation of PI 3-kinase signalling via inactivation of PTEN. EMBO J 2003, 22:5501-5510.

20. Yu CX, Li S, Whorton AR: Redox regulation of PTEN by S-nitrosothiols. Mol Pharmacol 2005, 68:847-854.

21. Covey TM, Edes K, Fitzpatrick FA: Akt activation by arachidonic acid metabolism occurs via oxidation and inactivation of PTEN tumor suppressor. Oncogene 2007, 26:5784-5792.

22. Lee SR, Yang KS, Kwon J, Lee C, Jeong W, Rhee SG: Reversible inactivation of the tumor suppressor PTEN by H2O2. J Biol Chem 2002, 277:20336-20342.

23. Kwon J, Lee SR, Yang KS, Ahn Y, Kim YJ, Stadtman ER, Rhee SG: Reversible oxidation and inactivation of the tumor suppressor PTEN in cells stimulated with peptide growth factors. Proc Natl Acad Sci USA 2004, 101:16419-16424.

24. Foster MW, Hess DT, Stamler JS: Protein S-nitrosylation in health and disease: a current perspective. Trends Mol Med 2009, 15:391-404.

25. Haendeler J, Hoffmann J, Tischler V, Berk BC, Zeiher AM, Dimmeler S: Redox regulatory and anti-apoptotic functions of thioredoxin depend on Snitrosylation at cysteine 69. Nat Cell Biol 2002, 4:743-749.

26. Chen L, Xu B, Liu L, Luo Y, Yin J, Zhou H, Chen W, Shen T, Han X, Huang S: Hydrogen peroxide inhibits mTOR signaling by activation of AMPKalpha leading to apoptosis of neuronal cells. Lab Invest 2010, 90:762-773.

27. Cai Z, Semenza GL: PTEN activity is modulated during ischemia and reperfusion: involvement in the induction and decay of preconditioning. Circ Res 2005, 97:1351-1359. 
28. Gidday JM: Cerebral preconditioning and ischaemic tolerance. Nat Rev Neurosci 2006, 7:437-448.

29. Zhao H: Ischemic postconditioning as a novel avenue to protect against brain injury after stroke. J Cereb Blood Flow Metab 2009, 29:873-885.

30. Calabrese V, Cornelius C, Rizzarelli E, Owen JB, Dinkova-Kostova AT, Butterfield DA: Nitric oxide in cell survival: a janus molecule. Antioxid Redox Signal 2009, 11:2717-2739.

31. Smith MA, Zhu X, Tabaton M, Liu G, McKeel DW Jr, Cohen ML, Wang X, Siedlak SL, Dwyer BE, Hayashi T, Nakamura M, Nunomura A, Perry G: Increased iron and free radical generation in preclinical Alzheimer disease and mild cognitive impairment. J Alzheimers Dis 2010, 19:363-372.

32. Lee JO, Yang H, Georgescu MM, Di Cristofano A, Maehama T, Shi Y, Dixon JE, Pandolfi P, Pavletich NP: Crystal structure of the PTEN tumor suppressor: implications for its phosphoinositide phosphatase activity and membrane association. Cell 1999, 99:323-334

33. Ross AH, Gericke A: Phosphorylation keeps PTEN phosphatase closed for business. Proc Natl Acad Sci USA 2009, 106:1297-1398.

34. Yao D, Gu Z, Nakamura T, Shi ZQ, Ma Y, Gaston B, Palmer LA, Rockenstein EM, Zhang Z, Masliah E, Uehara T, Lipton SA: Nitrosative stress linked to sporadic Parkinson's disease: S-nitrosylation of parkin regulates its E3 ubiquitin ligase activity. Proc Natl Acad Sci USA 2004, 101:10810-10814.

35. Kim S, Wing SS, Ponka P: S-nitrosylation of IRP2 regulates its stability via the ubiquitin-proteasome pathway. Mol Cell Biol 2004, 24:330-337.

36. Lee CM, Kim BY, Li L, Morgan ET: Nitric oxide-dependent proteasomal degradation of cytochrome P450 2B proteins.J Biol Chem 2008, 283:889-898.

37. Iyer AK, Azad N, Wang L, Rojanasakul Y: Role of S-nitrosylation in apoptosis resistance and carcinogenesis. Nitric Oxide 2008, 19:146-151.

38. Chanvorachote P, Nimmannit U, Lu Y, Talbott S, Jiang BH, Rojanasakul Y: Nitric oxide regulates lung carcinoma cell anoikis through inhibition of Ubiquitin-proteasomal degradation of caveolin-1. J Biol Chem 2009, 284:28476-28484.

39. Takashima A: GSK-3 is essential in the pathogenesis of Alzheimer's disease. J Alzheimers Dis 2006, 9(3 Suppl):309-317.

40. Kim GS, Jung JE, Niizuma K, Chan PH: CK2 is a novel negative regulator of NADPH oxidase and a neuroprotectant in mice after cerebral ischemia. $J$ Neurosci 2009, 29:14779-14789.

41. Liu F, Grundke-lqbal I, Iqbal K, Gong CX: Contributions of protein phosphatases PP1, PP2A, PP2B and PP5 to the regulation of tau phosphorylation. Eur J Neurosci 2005, 22:1942-1950.

42. Steen E, Terry BM, Rivera EJ, Cannon JL, Neely TR, Tavares R, Xu XJ, Wands JR, de la Monte SM: Impaired insulin and insulin-like growth factor expression and signaling mechanisms in Alzheimer's disease-is this type 3 diabetes? J Alzheimers Dis 2005, 7:63-80.

43. Pei JJ, Khatoon S, An WL, Nordlinder M, Tanaka T, Braak H, Tsujio I, Takeda M, Alafuzoff I, Winblad B, Cowburn RF, Grundke-lqbal I, Iqbal K: Role of protein kinase B in Alzheimer's neurofibrillary pathology. Acta Neuropathol 2003, 105:381-392.

44. Mocanu MM, Yellon DM: PTEN, the Achilles' heel of myocardial ischaemia/reperfusion injury? Br J Pharmacol 2007, 150:833-838.

45. Nagoshi T, Matsui T, Aoyama T, Leri A, Anversa P, Li L, Ogawa W, del Monte F, Gwathmey JK, Grazette L, Hemmings BA, Kass DA, Champion HC, Rosenzweig A: PI3K rescues the detrimental effects of chronic Akt activation in the heart during ischemia/reperfusion injury. $J$ Clin Invest 2005, 115:2128-2138.

46. Wang CY, Kim HH, Hiroi Y, Sawada N, Salomone S, Benjamin LE, Walsh K, Moskowitz MA, Liao JK: Obesity increases vascular senescence and susceptibility to ischemic injury through chronic activation of Akt and mTOR. Sci Signal 2009, 2(62):ra11.

47. Fraser MM, Bayazitov IT, Zakharenko SS, Baker SJ: Phosphatase and tensin homolog, deleted on chromosome 10 deficiency in brain causes defects in synaptic structure, transmission and plasticity, and myelination abnormalities. Neuroscience 2008, 151:476-488.

48. Ma T, Zhao Y, Kwak YD, Yang Z, Thompson R, Luo Z, Xu H, Liao FF: Statin's excitoprotection is mediated by SAPP and the subsequent attenuation of calpain-induced truncation events, likely via rho-ROCK signaling. $J$ Neurosci 2009, 29:11226-11236

49. Lei SZ, Pan ZH, Aggarwal SK, Chen HS, Hartman J, Sucher NJ, Lipton SA: Effect of nitric oxide production on the redox modulatory site of the NMDA receptor-channel complex. Neuron 1992, 8:1087-1099.
50. Jaffrey SR, Synder SH: The biotin switch method for the detection of Snitrosylated proteins. Sci STKE 2001, 2001:pl1.

51. Podsypanina K, Ellenson LH, Nemes A, Gu J, Tamura M, Yamada KM, Cordon-Cardo C, Catoretti G, Fisher PE, Parsons R: Mutation of Pten/ Mmac1 in mice causes neoplasia in multiple organ systems. Proc Natl Acad Sci USA 1999, 96:1563-1568.

doi:10.1186/1750-1326-5-49

Cite this article as: Kwak et al:: NO signaling and S-nitrosylation regulate PTEN inhibition in neurodegeneration. Molecular Neurodegeneration 2010 5:49.

\section{Submit your next manuscript to BioMed Central and take full advantage of:}

- Convenient online submission

- Thorough peer review

- No space constraints or color figure charges

- Immediate publication on acceptance

- Inclusion in PubMed, CAS, Scopus and Google Scholar

- Research which is freely available for redistribution
C Biomed Central 